\title{
The progress in repair research of ancient timber structure
}

\author{
Li $\operatorname{sisi}^{1, *}$, Zhou xun ${ }^{1}$ \\ ${ }^{1}$ School of Civil Engineering an Architecture, Southwest University of Science and Technology, Mianyang, Sichuan 621010
}

\begin{abstract}
Ancient architecture carries Chinese culture, which is helpful to understand the tradition. Timber structure is the main structure of ancient Chinese buildings, and most of the ancient timber structures have been overserved and have been damaged to varying degrees. Although timber structure is made of convenient materials, its low strength and vulnerability to corrosion increase the difficulty of the protection of ancient buildings, so the restoration of ancient buildings is imminent. Through literature research, this paper summarized the repair principles and reinforcement methods, and analyzed the effective methods and research progress of the repair and reinforcement of ancient timber structures from the traditional and modern aspects, and provided reference for the repair work of ancient timber structures.
\end{abstract}

\section{Introduction}

Compared with the western ancient architecture. Chinese ancient architecture has its unique forms, styles and structures, such as brackets, mortise and tenon, etc., so it has also attracted the attention of many researchers. Wood structure, as the mainstream of Chinese ancient architecture, takes wood as load-bearing component and has good seismic capacity. However, with the occurrence of man-made damage and natural disasters, plus the wood itself being easy to corrode, moth-eaten, most of the existing ancient buildings have been damaged to varying degrees.

The East Hall of Foguang Temple, located in Wutai County, Shanxi Province, is the largest and only remaining timber structure of the Tang Dynasty in China. In 2017, the main hall leaked rain, and it was roughly estimated that there were at least a dozen raindrops. In fact, in 2015, experts have been debating whether there is a better way to protect it. The same thing happened when the Yingxian wooden tower was repaired. Even though there were a lot of schemes, they still dared not act rashly. At present, many ancient buildings that have been repaired and reinforced are maintained in accordance with traditional reinforcement methods. But obviously, most of the existing studies on the maintenance and reinforcement of ancient timber structures can not meet the needs of practical engineering.

Based on the existing research, this paper mainly introduces, analyzes and compares the traditional experience and the new maintenance and reinforcement methods, summarizes the current situation, and looks forward to the future research and development trend.

\section{2 the principles for the repair of ancient buildings}

\subsection{Principle of preservation of cultural relics}

Original state refers to all the status of historical significance in the individual or group of ancient buildings ${ }^{[1]}$. While maintaining the ancient buildings, the original architectural form (plane layout, modeling, artistic style, etc.), the original architectural structure, the original building materials and technology should be retained. When we repair it, we must not change its original state, otherwise it is man-made damage, but also according to the relevant historical and technical information to protect it.

\subsection{The principle of "make the old as the old"}

In the 20th century, Liang Sicheng became the representative of some scholars, put forward the concept of "the old as the old". According to the Technical Code for Maintenance and Strengthening of ancient timber structures, the reliability of structures should be evaluated according to the calculated damage points in the load-bearing structures, which coincides with the principle of "the old as the old". "The old as the old" should give people the feeling of "being old and strong", rather than "rejuvenating" [2].

All in all, the repair process of ancient buildings is to make the ancient buildings glow with historical aesthetic $^{[3]}$. The restoration work should be combined with the idea of sustainable development, which should not only preserve the original appearance of the ancient building, but also reserve space for the future repair work. 


\section{Traditional reinforcement and maintenance methods}

\subsection{Inlay reinforcement method}

The column may have some cracks due to the nature of the wood itself. When the column radius or $1 / 3$ of the section size exceeds the depth of the crack, the column may be reinforced by the method of nesting: When the width of the crack is less than the specified value, the surface layer can be filled by painting or oil decoration and so on. When crack width is larger, using wood strips and water-resistant adhesive to have filling. The patent ${ }^{[4]}$ improves this method with fast construction progress, low cost and high safety, and only repairs the damaged parts, which can retaining the original appearance of the damaged components to a large extent.

\subsection{Pier reinforcement method}

The scope of application is the part of the decayed column foot and the length of the damage along the bottom of the column is less than 1/4 of the height of the wooden column. Pier connection is divided into the following three kinds: wood pier joint construction must strictly control the accurate docking of pier tenon, and iron parts should also be set at the joint; Reinforced concrete pier connection is generally only used in the wall of the hidden column, the height and diameter of the column also has the corresponding specification requirements; Stone pier connection, its size should be smaller than the original column diameter $100 \mathrm{~mm}$, and the application of plank and iron hoop will be wrapped and fixed. In 1999, the pier joint method was applied and improved in the restoration and reinforcement of the corner eave columns of Tian An Men. So far, no problems have been found ${ }^{[5]}$.

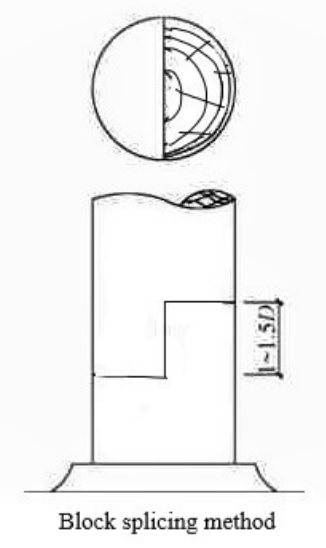

Fig. 1 Block splicing method

\subsection{Propping reinforcement method}

This method is mainly to reduce the deflection and bending moment of the beam frame by propping. The main principle is to use wooden column or iron hook as the external support of the beam frame member, so that the internal force is redistributed and the bearing capacity of the member is further improved. The Yingxian wooden pagoda in Shanxi Province adopted the method of erecting new components. From the present situation, most of the additional components of the wooden pagoda have played a good role ${ }^{[6]}$.

\subsection{Iron reinforcement method}

When the beam and column produce large cracks, in addition to the inserting and wiping, but also need to use iron part to reinforce it; When the scope of decay is large, it is also necessary to adopt iron reinforcement for the members with pier reinforcement. This method mainly relies on the restraint effect of iron to improve the strength and stiffness of the members. Zhou Gan et al. ${ }^{[7]}$ put forward appropriate suggestions by analyzing the application of iron reinforcement in various projects such as the Hall of Supreme Harmony of the Imperial Palace and the wooden pagoda in Yingxian County.

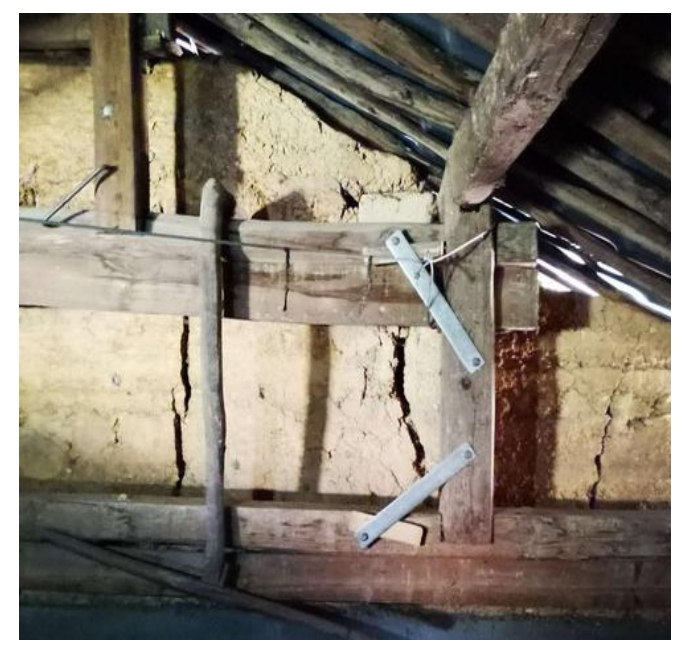

Fig. 2 Iron reinforcement

To sum up, the traditional reinforcement method can improve the bearing capacity and stability of wood structure to a certain extent, but there are also some problems that need to be improved:

(1) Some repair measures can only temporarily maintain ancient buildings, but may not be effective in the long run. For example because of the outstanding creep performance of wood is more prominent, iron is easy to deformation and rust; Moreover, the traditional iron reinforcement method adopts iron material, which is easy to lead to the cracking of the components. In the long run, it will not only destroy the material, but also cause new problems.

(2) Propping reinforcement, iron reinforcement and other reinforcement methods will damage the building appearance to a certain extent, and there is a certain irreversibility, so that the subsequent repair work is not easy to carry out. 


\section{New reinforcement and repair materials and technology}

Compared with the traditional reinforcement and restoration technology, the new technology may better meet actual requirements and improve the quality of repair, without changing the original appearance of the ancient building. At present, a large number of new technologies used in practical engineering include chemical reinforcement, prestressed reinforcement technology, new materials include Carbon Fibre-reinforced Polymer, glass reinforced plastics and basalt fiber.

\subsection{New materials}

\section{(1) Carbon Fibre-reinforced Polymer (CFRP)}

Carbon fiber reinforced material is a composite material with carbon fiber as the reinforcements, in addition, there are resin, ceramic, carbon and so on as the matrix. This material has high temperature resistance, stable shape, shock absorption, radiation resistance, higher strength and lighter weight than other materials, which can effectively guarantee the maintenance and reinforcement quality of timber structure. At the same time, the penetration adhesive should be used in combination with CFRP. The penetration adhesive is mainly used for the repair of timber structure cracks and anti-aging work, and it should be brushed on the surface of carbon fiber to enhance its durability ${ }^{[8]}$. The use of CFRP can effectively improve the stiffness and increase the failure load of wood components ${ }^{[9]}$.

(2) Glass reinforced plastics (GFRP)

Glass fiber reinforced material is also a kind of composite plastics, with glass fiber reinforced unsaturated polyester and a variety of polymer compounds as the matrix. It has many similar advantages with CFRP, such as light weight, high strength, corrosion resistance and so on. However, CFRP has poor mechanical properties in the repair of square cross-section members, which often leads to waste of materials, while GFRP has better help in the repair of square section of timber structure, which can significantly improve the durability of the structure and avoid waste of materials ${ }^{[10]}$.

\section{(3) Basalt fiber (CBF)}

Basalt fiber is a kind of high strength fiber formed by drawing natural basalt, and has a variety of properties that other materials lack, such as insulation, corrosion resistance, high temperature resistance and so on. The production process has little pollution to the environment, and the products can be directly degraded in the natural environment after use. Although its elastic modulus is not as good as other materials, it is better than carbon fiber in the affinity with resin, which can prolong the reinforcing effect and the service life of the reinforcing material ${ }^{[11]}$. Basalt fiber is easy to cut, plasticity is strong. The damage to the appearance of timber structure is very small, and the operation is also easy to implement.

\subsection{Chemical reinforcement}

Chemical reinforcement method is generally used in hollow or decayed in wood interior, clear the decayed part thoroughly first, reoccupy unsaturated get together the chemical reagents such as resin undertakes perfuse, the chemical reagent that uses also should accord with relevant regulation. According to [12], the pillar of Ximatai Fengyunge of Xuzhou was repaired by chemical reinforcement method, and the effect was good. In general, chemical reinforcements are used in conjunction with CFRP, GFRP, and CBF to achieve better restoration.

\subsection{Prestressed reinforcement}

The prestressed reinforcement usually takes the additional draw bar or the support rod to strengthen the component. Before the force, a certain compressive stress can be applied to the component in advance, so that part of the tensile stress can be offset when bearing the load, and the deformation and vibration of the component are constrained while the bearing capacity of the component is improved. When using the prestressing technology to strengthen the timber structure, the carbon fiber cloth is glued to the tensile area to generate tensile stress, so that the overall timber structure can prestressed, so as to improve the bearing capacity and stiffness of members [13].

\section{General Research Status}

In recent years, experts have carried out research and discussion on ancient timber structures in different aspects, and achieved certain results. Xie Qi-fang et al. ${ }^{[14]}$ studied the seismic resistance of the damaged one-way straight tenon joints, and the test results showed that the rotational bending moment, rotational stiffness and energy dissipation capacity of the damaged joints decreased with the increase of the degree of damage, but they still had good ductility. He Jun-xiao et al. ${ }^{[15]}$ analyzed the mechanical property, hysteretic performance, rotational stiffness and other characteristics of column foot joints under low-cyclic loading, and obtained the coefficient of vertical load restoring bending moment and rotational stiffness. Xue Jian-yang et al. ${ }^{[16]}$ gave the equivalent model of each component and put forward the dynamic analysis calculation model of the ancient timber building with monolayer hall-style structure. Zhang Feng-liang et al. ${ }^{[17]}$ calculated the formula for calculating the flexural capacity of the damaged joints of timber structures strengthened with CFRP, and gave appropriate reinforcement suggestions. Shang Tao et al. ${ }^{[18]}$ used close-range photogrammetry and 3-D laser scanning technology to establish the model, take the ancient buildings of Sizu Temple and Wuzu Temple in Hubei Province for example, and recover them in proportion by rapid prototyping. Ma Hong-yu et al. ${ }^{[19]}$ proposed a method of combining BIM technology and 3D laser scanning technology, and created 3D models of ancient buildings, mapping data and information database through this method. Zhang Feng-liang et al. ${ }^{[20]}$ 
established the finite element model of roof-beams frame system and carried out dynamic analysis on it, and obtained the research results to provide help for the study of seismic performance of ancient wooden structures.

\section{Conclusion}

To sum up, the ancient timber structure should follow the repair principle of preserving the original state and "the old as the old", so as to preserve its cultural and historical background. However, the traditional reinforcement method will cause different degrees of damage to the appearance of the ancient building and the material itself. With the emergence of new technology and new materials, many new reinforcement methods, such as CFRP and chemical reinforcement, have been gradually used in the repair of ancient timber structures. When reinforcing, we should be clear about the applicable scope and limitation of different methods, and adopt appropriate reinforcement methods according to different failure symptoms.

In the future, more new materials and technologies can be used in the repair, using more environmentally friendly and energy-saving materials, and the secondary or multiple reinforcement of the ancient timber structure can be reduced; Moreover, the new technology can be combined with the traditional reinforcement technology to make up for the shortcomings of the two, saving costs and protecting the historic sites while reducing the damage to their original appearance.

Among all building materials, wood is the only renewable resource ${ }^{[21]}$. The building built with wood is seismic, safety, heat preservation, energy saving, and the development of timber structure is of great help to the improvement of the environment. Modern timber structure can learn from the technology of ancient timber structure to get better development

\section{Reference:}

1. GB 50165-1992.Technical Code for Maintenance and Strengthening of Ancient Timber Structures. S.

2. Liang S.C. (1963) Talk about the restoration and maintenance of historic relics. J. Cultural Relics, 07: 5-10.

3. Qi W.C. (2011) Principles and techniques of restoration of ancient Chinese buildings. J. Construction and Architecture, 11: 27-28.

4. Che W.S, Huang W.C, Huang L, Jin Y.S. Method for repairing wood beam column of old building by embedded rib reinforcement. P. Sichuan: CN101922241A, 2010-12-22.

5. Zhang F.L. (2004) Research and construction of joint technology of column and pier of corner eaves of tiananmen gatehouse. J. Traditional Chinese Architecture and Gardens, 02: 51-53+12.

6. Li T.Y, Qin H.M. (2005) Structural analysis and repair of Yingxian wooden tower. J. Engineering Mechanics, S1: 199-212.
7. Zhou Q, Yan W.M. (2011) Application of Iron-strengthening Techniques in Chinese Ancient Buildings. J. Journal of Water Resources and Architectural Engineering, 9(01): 1-5+61.

8. Luo C.S, Huang Y.P. (2005) The Analysis on the Traditional Reinforced Methods of Ancient Timber Building and the Discussing on the Application of FRP in the Field. J. Fujian Architecture and Construction, Z1: 208-210+213.

9. Liu X.Y, Cheng Y.S. (2003) The discussion about the protection of ancient timber structures from the treatment of anti-corrosion and insect control of Xingguo Temple. J. Traditional Chinese Architecture and Gardens, 04: 44-47.

10. Zhou Q.L. (2019) Application of new materials in restoration of ancient timber buildings. J. Technology and Economic Guide, 27(13): 51-52.

11. Zhou Qian, Yan Wei-ming, Li Zhen-bao, Ji jin-bao. (2009) Study on Strengthening Methods of Timber Structures of Ancient Buildings. J. Earthquake Resistant Engineering and Retrofitting, 31(01): 84-90.

12. Ma J.L, Bai R.S, Cai H.Q. (2007) Research and Application of Timber Structure Reinforcement with CBFRPS. J. Architecture Technology, 06: 426-427.

13. Xu X.J. (1999) A brief discussion on the maintenance of ancient building wood components. J. Traditional Chinese Architecture and Gardens, 01: 42.

14. Xie Q.F, Zheng P.J, Xiang W, Cui Y.A, Zhang F.L. (2014) Experimental study on seismic behavior of damaged straight mortise-tenon joints of ancient timber buildings. J. Journal of Building Structures, 35(11): 143-150.

15. He J.X, Wang J, Yang Q.S. (2017) Mechanical property of column footing joint in traditional wooden structure by quasi-static test. J. Journal of Building Structures, 38(08): 141-149.

16. Xue J.Y, Zhang F.L, Zhao H.T, et al. (2012) Dynamic analysis odel of monolayer hall-style ancient timber structure. J. Journal of Building Structures, 33(08): $135-142$.

17. Zhang F.L, Lu J.Y, Xue J.Y, et al. (2015) Performance analysis and design recommendations for damaged mortise-tenon joint of ancient timber structures strengthened with CFRP. J. Journal of Building Structures, 45(06): 61-65+13.

18. Shang T, Kong L.M. (2006) Research on theprotected method ofancient building by digitaltechnology. J. Engineering Journal of Wuhan University, 01: 72-75.

19. Ma H.Y, Zhao X. (2019) 3D Laser Scanning Technology and BIM Technology in Ancient Building Protection Surveying and Mapping. J. Geotechnical Engineering Technique, 33(04): 222-225.

20. Zhang F.L, Zhao H.T, Xue J.Y, et al. (2012) The Analysis of Dynamic Properties on The Roof-Beams 
System in Ancient Timber Buildings. J. Engineering Mechanics, 29(08): 184-188+201.

21. Lou W.L, Ren H.Q. (2015) Characteristics and Prospect of Wood Structure Buildings in China. J. China Wood Industry, 29(05): 20-23. 